\title{
A role for IL-17 in age-related macular degeneration
}

\author{
Jae II Shin and Jagadeesh Bayry
}

In a recent article ${ }^{1}$, Ambati and colleagues discussed the immunological aspects of age-related macular degeneration (AMD) pathogenesis and inflammation-directed therapeutics to treat AMD.

Based on the current literature and evidence, they deliberated that AMD could be caused by various factors, such as immunemediated retinal damage (for example, interleukin-1 $\beta$ (IL-1 $\beta$ ), IL-6 and IL-18), the pro-inflammatory components of drusen (for example, $\beta$-amyloid), complement activation (for example, C1q, C3 and C5 activation), proliferative angiogenic responses causing neovascularization (for example, IL-1 $\beta$, IL-6, tumour necrosis factor (TNF) and vascular endothelial growth factor $\mathrm{A}$ (VEGFA)), and geographic atrophy caused by prolonged VEGFA-specific antibody therapy ${ }^{1}$. However, we would like to add that the IL-17 signalling pathway is also likely to be important in the pathogenesis of AMD.

IL-17 is a signature cytokine of the T helper $17\left(\mathrm{~T}_{\mathrm{H}} 17\right)$ cell subset and has a crucial role in promoting inflammation in various autoimmune and inflammatory diseases ${ }^{2}$. In addition to $\mathrm{T}_{H} 17$ cells, $\gamma \delta \mathrm{T}$ cells and innate lymphoid cells (ILCs) also produce IL-17. Recently, several reports have demonstrated the involvement of IL-17 in the pathogenic inflammation of $\mathrm{AMD}^{3-5}$. Liu et al. ${ }^{3}$ reported that complement component $5 \mathrm{a}$ (C5a) is increased in the circulation of AMD patients and that it promotes IL-17 and IL-22 expression by human $\mathrm{CD} 4^{+} \mathrm{T}$ cells. These authors found significantly elevated levels of IL-17 and IL-22 in patients with AMD compared with control individuals who did not have AMD. Furthermore, in laser-induced experimental choroidal neovascularization - which has characteristic features of AMD - Hasegawa et al. ${ }^{4}$ showed that IL-17 has a strong potential for stimulating neovascularization in a VEGF-independent manner. Importantly, the authors reported that $\gamma \delta$ T cells and THY $1^{+}$ILCs, but not $\mathrm{T}_{\mathrm{H}} 17$ cells, were the relevant source of IL-17. In line with the findings of Liu et al. ${ }^{3}, \mathrm{IL}-1 \beta$ in combination with high-mobility group box 1 (HMGB1) induced IL-17 production by $\gamma \delta$ T cells. Tuo et al. ${ }^{5}$ demonstrated that intravitreous administration of recombinant TNF-inducible gene 6 protein (TSG6) could stabilize retinal lesions in mice that are deficient in both CC-chemokine ligand 2 (CCL2) and $\mathrm{CX}_{3} \mathrm{C}$-chemokine receptor 1 $\left(\mathrm{CX}_{3} \mathrm{CR} 1\right)$ by modulating the expression of several ocular immunological genes and, in particular, Il17a. IL-17 is also known to promote VEGF-mediated angiogenesis by enhancing VEGF-induced growth of vascular endothelial cells ${ }^{6}$.

All of these reports point towards the possibility that IL-17 could be involved in the pathogenesis of AMD by promoting retinal angiogenesis and neovascularization. Therefore, as a proof-of-concept, IL-17-targeted therapies could be explored, at least in experimental models.

Jae II Shin is at the Department of Pediatrics, Yonsei University College of Medicine, Seoul 120-752, Korea

Jagadeesh Bayry is at the Institut National de la Santé et de la Recherche Médicale, Unité 872, Paris

F-75006, France; at the Centre de Recherche des Cordeliers, Equipe 16-Immunopathology and Therapeutic Immunointervention, Université Paris 6 Pierre et Marie Curie, Paris F-75006, France; and at the Université Paris Descartes, UMR S 872, 15 rue de l'Ecole de Médicine, Paris F-75006, France.

e-mails: pedshin2000@yahoo.co.kr jagadeesh.bayry@crc.jussieu.fr

1. Ambati, J. et al. Immunology of age-related macular degeneration. Nature Rev. Immunol. 13, 438-451 (2013).

2. Gaffen, S. L. Structure and signalling in the IL-17 receptor family. Nature Rev. Immunol. 9, 556-567 (2009).

3. Liu, B. et al Complement component C5a promotes expression of IL-22 and IL-17 from human T cells and its implication in age-related macular degeneration. J. Transl. Med. 9, 111 (2011).

4. Hasegawa, E. et al. IL-23-independent induction of IL-17 from $\gamma \delta$ T cells and innate lymphoid cells promotes experimental intraocular neovascularization. J. Immunol. 190, 1778-1787 (2013).

5. Tuo, J. et al. Anti-inflammatory recombinant TSG-6 stabilizes the progression of focal retinal degeneration in a murine model. J. Neuroinflammation 9, 59 (2012).

6. Takahashi, H. et al. Interleukin-17 enhances bFGFHGF- and VEGF-induced growth of vascular endothelial cells. Immunol. Lett. 98, 189-193 (2005).

Competing interests statement

The authors declare no competing financial interests. 